\title{
An innovative global cloudless satellite map
}

\author{
Nicolas Bozon ${ }^{\mathrm{a}, *}$, Petr Pridal ${ }^{\mathrm{a}}$, Martin Koštál ${ }^{\mathrm{a}}$ \\ aMapTiler,nicolas.bozon@maptiler.com,petr.pridal@maptiler.com,martin.kostal@maptiler.com \\ * Corresponding author
}

Keywords: Satellite imagery, Cloudless, Deep learning, Color toning, High-resolution, Orthophotography, Open data

\section{Abstract:}

A new cloudless global satellite map is presented as the result of an innovative research and development project. An Innovative open data processing pipeline is first described and the resulting value-added product is then detailed through example use-cases.

Important volumes of open satellite imagery available from the ESA Copernicus program are being sorted and selected with dedicated deep learning algorithms. The latter were trained according to multiple spatial, temporal and cloud coverage parameters and allowed to fetch the most suitable Sentinel-2 L2A images in a very effective way. Summer time series were programmatically explored for both hemispheres, and millions of candidate images were sourced across the Earth's emerged lands. 180 Terabytes of images were assembled together into a global composite map and their pixels harmonized using a unique color-toning technique. Red, green and blue channels are automatically adjusted according to desired specific colors. MapTiler Satellite thus optimizes the Earth natural colors rendering as seen by the human eye.

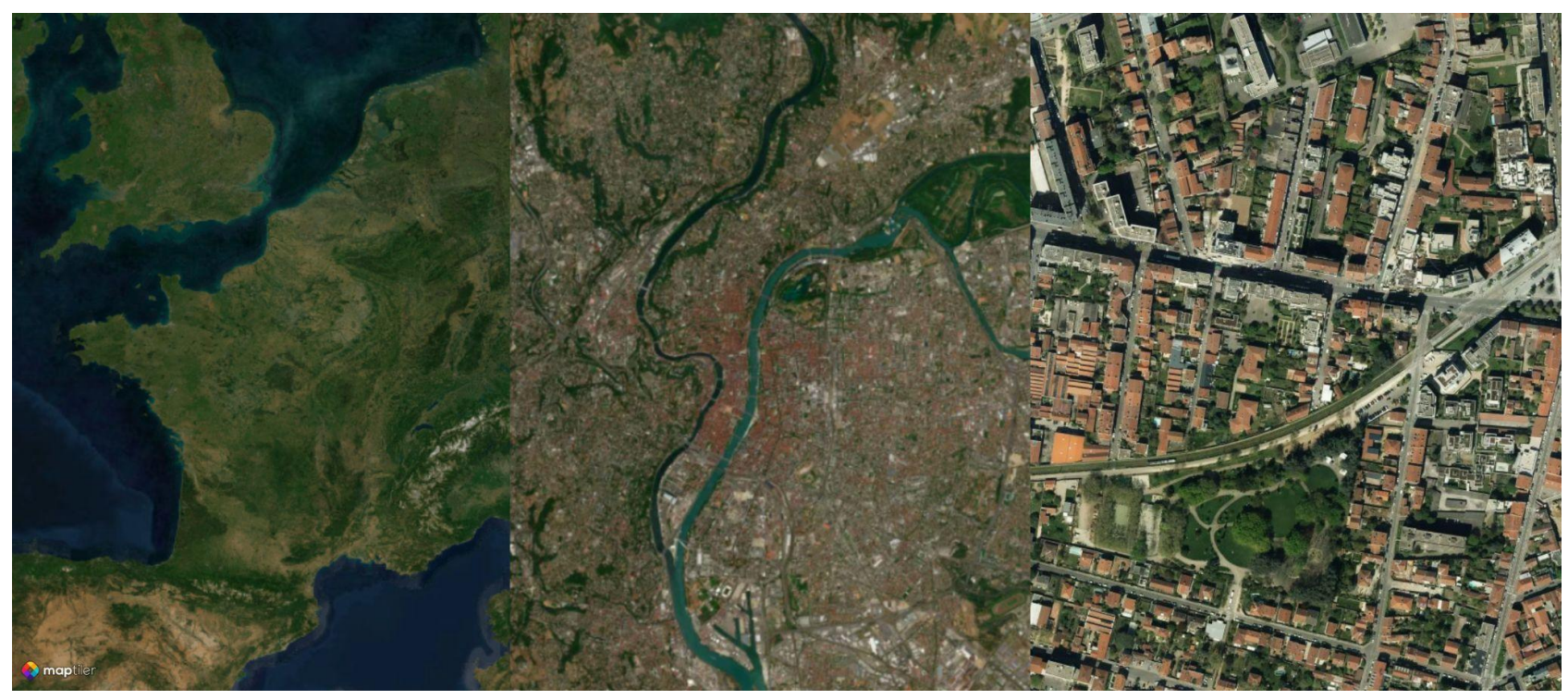

Figure 1. Preview of the MapTiler Satellite map at small, medium and large scales

The resulting color-toned 10m per pixel imagery is finally generated according to a global WGS84 regular grid and a web mercator tiling scheme, and produced as both GeoTIFF and ready-to-use map tiles. The Satellite map is supplemented by very high-resolution aerial imagery (50 to $20 \mathrm{~cm} /$ pixels) for regions where it is released as open data by national or local governments. The United States, Japan and most of Western Europe are already available across all zoom levels, and more regions are being added to the map according to the new open imagery releases worldwide.

The whole processing and color-toning pipeline can be set up for different types of imagery and available through MapTiler consulting services. The result of this work is used to produce a qualitative high-resolution satellite basemap served from MapTiler's cloud infrastructure, where it can be modified and used in conjunction with OpenStreetMap vector tiles and other datasets. The map is also made available through the MapTiler Data and MapTiler Server products for self-hosting and offline use-cases. MapTiler Satellite is thus used in numerous GIS, Web and mobile applications and viewed daily by millions of users. 